\title{
Using Facebook to Announce the Latest Glyco-related Research Articles
}

This year, FCCA started announcing the latest "glyco"-related articles released by publishers via Facebook. Carbohydrates are physically, biologically, and conceptually combined with other biomolecules and interact with their environment so that they cannot be often treated independently. This results in many carbohydrate-related research articles are published in a number of more general journals in various fields, which further makes access difficult. With our hope that a site introducing recently published papers would help researchers and students, we initiated this Facebook page.

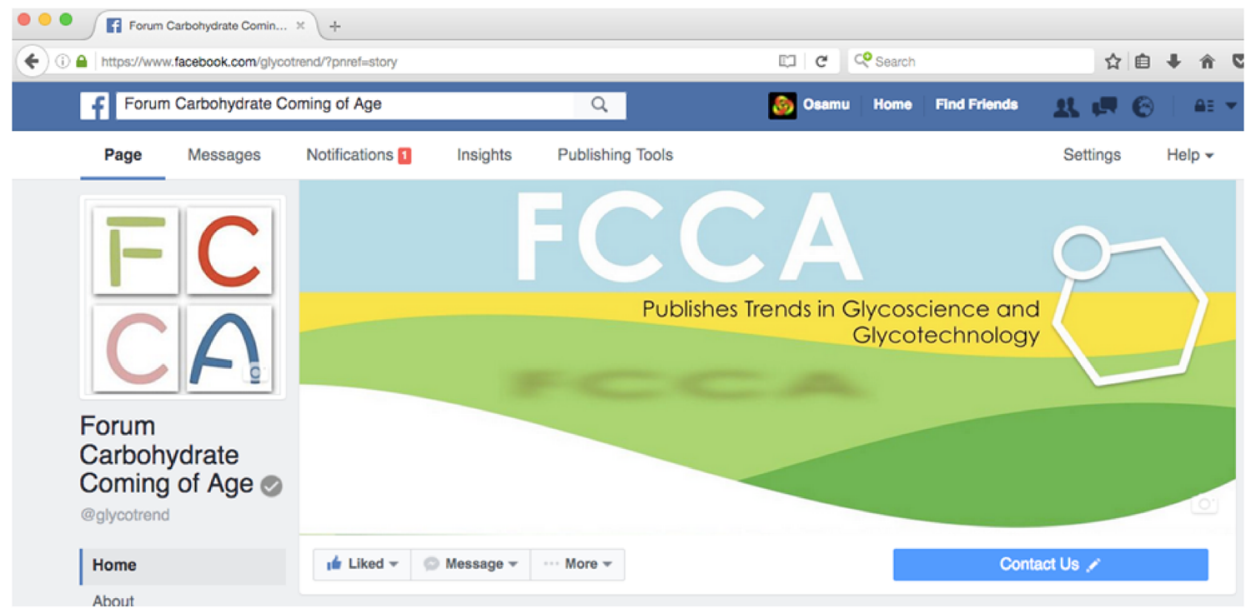

We named our page "Forum Carbohydrate Coming of Age," but initially it was difficult or almost impossible to find on the Web by searching through search engines. Thus, we applied a nickname "glycotrend" so that readers can find our page by searching for either "forum carbohydrate coming of age" or "glycotrend."

As FCCA is a Japan-based non-profit group, we started this page in Japanese basically because of the tendency of Japanese to hesitate to access English pages despite the fact that we even know it is unavoidable to face English. We probably live in a country that makes it too easy for us to avoid English on a daily basis. This put us behind from the rest of the world, and so of course we should deal with this fact. However, since we tend to avoid facing such realities and instead tend to deal with the closer reality inside our country, we at FCCA decided to start this experiment in our own tongue but dealing with information from around the world. We hope this will prompt all of us to better face the world. We also hope, for now, that translation tools allow people from around the world to access our posts as well.

As a matter of fact, Google's translation service translates the content of our posts into more than 80 languages. Access analysis showed that people from many countries had reached our posts and further clicked on the article introduced. By analyzing the coun-

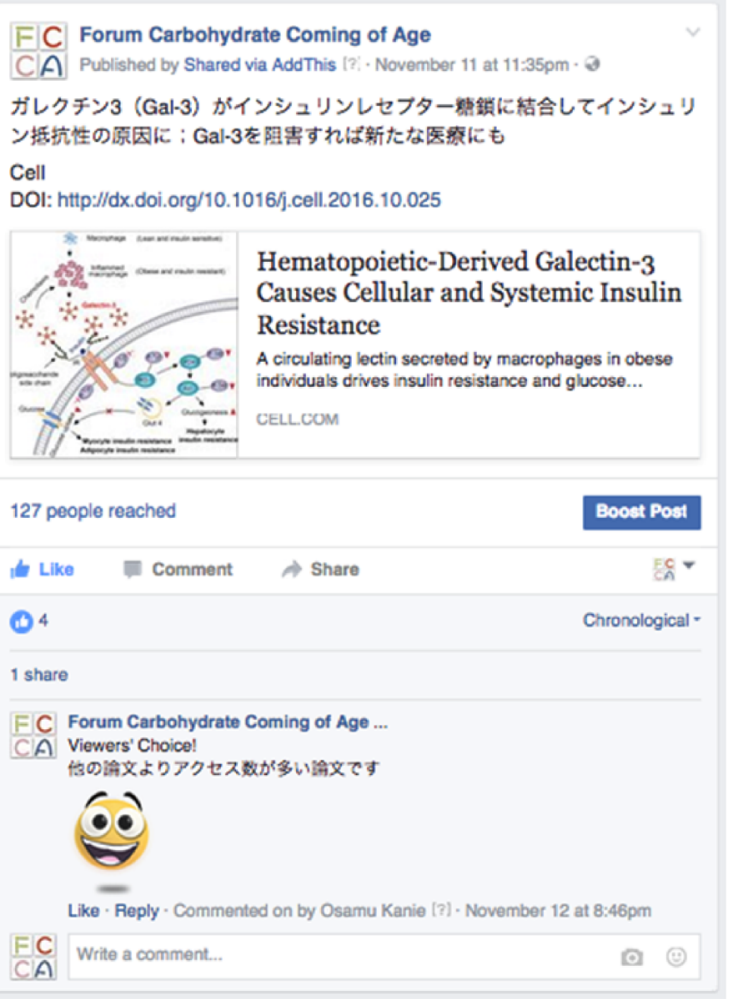




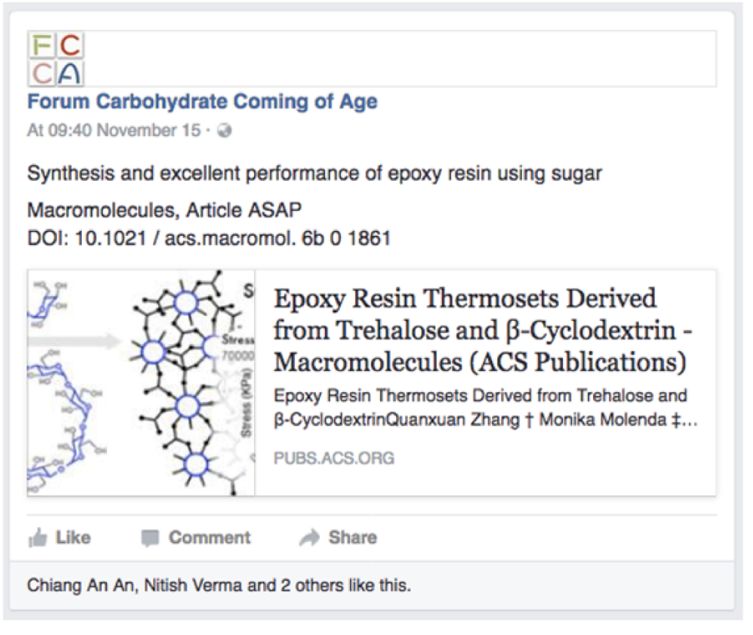

tries from which readers made access, we were very pleased to find that people from around the world, including Japan of course (46\%), but also Taiwan (20\%), India (13\%), United States (8\%), Germany, Switzerland, Finland, United Kingdom, South Korea, China, Vietnam, Canada, Thailand, Spain, France, Sweden, Philippines, Denmark, Turkey, Australia, Belgium, Iran, Hong Kong, Portugal, Bangladesh, Indonesia, Singapore, Sudan, and Sri Lanka were reading our posts! If we look at people by age, readers could be broken down into the following groups, 18-24 (19\%), 25-34 (40\%), 35-44 (26\%), and 45-54 (9\%), which suggests fairness of our initial considerations.

We have posted more than 200 articles during the past six months. Among them, some has been paid considerably higher attention compared to others. These were highlighted as "viewers' choice" on our site and are listed below.

1. A catalytic role for $\mathrm{C}-\mathrm{H} / \pi$ interactions in base excision repair by Bacillus cereus DNA glycosylase AlkD, Z.D. Parsons, J.M. Bland, E.A. Mullins, B.F. Eichman, J. Am. Chem. Soc., doi: 10.1021/jacs.6b07399

2. A modular approach to a library of semi-synthetic fucosylated chondroitin sulfate polysaccharides with different sulfation and fucosylation patterns, A. Laezza, A. Iadonisi, A.V.A. Pirozzi, P. Diana, M. De Rosa, C. Schiraldi, M. Parrilli, E. Bedini, Chem. Eur. J., doi: 10.1002/chem.201603525

3. A novel chemoenzymatic synthesis of sulfated type 2 tumorassociated carbohydrate antigens by transglycosylation of sulfated Lewis X oxazoline catalyzed by keratanase II, Y. Yamazaki, K. Sezukuri, J. Takada, S. Kimura, M. Ohmae, ChemBioChem, doi: 10.1002/cbic.201600142

4. A reversible B-A transition of DNA duplexes induced by synthetic cationic copolymers, N. Yamaguchi, Y. Zouzumi, N. Shimada, S.-I. Nakano, N. Sugimoto, A. Maruyama, D. Miyoshi, Chem. Commun., doi: 10.1039/C6CC02237E
5. A synthetic biochemistry module for production of bio-based chemicals from glucose, P.H. Opgenorth, T.P. Korman, J.U. Bowie, Nat. Chem. Biol., doi: 10.1038/nchembio.2062

6. A visible-light-promoted O-glycosylation with a thioglycoside donor, M.L. Spell, K. Deveaux, C.G. Bresnahan, B.L. Bernard, W. Sheffield, R. Kumar, J.R. Ragains, Angew. Chem. Int. Ed., doi: 10.1002/anie.201601566

7. Atomic visualization of a flipped-back conformation of bisected glycans bound to specific lectins, M. Nagae, M. Kanagawa, K. Morita-Matsumoto, S. Hanashima, Y. Kizuka, N. Taniguchi, Y. Yamaguchi, Sci. Rep., doi: 10.1038/ srep 22973

8. Automated glycan sequencing from tandem mass spectra of N-linked glycopeptides, C.-Y. Yu, A. Mayampurath, R. Zhu, L. Zacharias, E. Song, L. Wang, Y. Mechref, H. Tang, Anal. Chem., doi: 10.1021/acs.analchem.5b04858

9. Bacteriophage tailspikes and bacterial O-antigens as a model system to study weak-affinity protein-polysaccharide interactions, Y. Kang, U. Gohlke, O. Engström, C. Hamark, T. Scheidt, S. Kunstmann, U. Heinemann, G. Widmalm, M. Santer, S. Barbirz, J. Am. Chem. Soc., doi: 10.1021/ jacs.6b00240

10. Basic amino acids as green catalysts for isomerization of glucose to fructose in water, Q. Yang, M. Sherbahn, T. Runge, ACS Sustainable Chem. Eng., doi: 10.1021/ acssuschemeng.6b00587

11. Building nanowires from micelles: Hierarchical self-assembly of alternating amphiphilic glycopolypeptide brushes with pendants of high-mannose glycodendron and oligophenylalanine, Y. Liu, Y. Zhang, Z. Wang, J. Wang, K. Wei, Guosong Chen, Ming Jiang, J. Am. Chem. Soc., doi: 10.1021/jacs.6b05044

12. Catching elusive glycosyl cations in a condensed phase with HF/SbF5 superacid, A. Martin, A. Arda, J. Désiré, A. Martin-Mingot, N. Probst, P. Sinaÿ, J. Jiménez-Barbero, S. Thibaudeau, Y. Blériot, Nat. Chem., doi: 10.1038/nchem.2399

13. Cellulose-based biosensors for esterase detection, F. Derikvand, D.T. Yin, R. Barrett, H. Brumer, Anal. Chem., doi: 10.1021/acs.analchem.5b04661

14. Chemical approach to positional isomers of glucose-platinum conjugates reveals specific cancer targeting through glucosetransporter-mediated uptake in vitro and in vivo, M. Patra, S.G. Awuah, S.J. Lippard, J. Am. Chem. Soc., doi: 10.1021/ jacs.6b06937

15. Chemical O-glycosylations: An overview, R. Das, B. Mukhopadhyay, Chem. Open, doi: 10.1002/open.201600043

16. Chemical synthesis of a complex-type N-glycan containing a core fucose, M. Nagasaki, Y. Manabe, N. Minamoto, K. 
Tanaka, A. Silipo, A. Molinaro, K. Fukase, J. Org. Chem., doi: 10.1021/acs.joc.6b02106

17. Convergent solid-phase synthesis of macromolecular MUC1 models truly mimicking serum glycoprotein biomarkers of interstitial lung diseases, N. Ohyabu, K. Kakiya, Y. Yokoi, H. Hinou, S.-I. Nishimura, J. Am. Chem. Soc., doi: 10.1021/ jacs.6b04973

18. Cracking the glycome encoder: Signaling, trafficking, and glycosylation, F. Bard, J. Chia, Trends Cell Biol., doi: 10.1016/j.tcb.2015.12.004

19. Development of rare bacterial monosaccharide analogs for metabolic glycan labeling in pathogenic bacteria, E.L. Clark, M. Emmadi, K.L. Krupp, A.R. Podilapu, J.D. Helble, S.S. Kulkarni, D.H. Dube, ACS Chem. Biol., doi: 10.1021/ acschembio.6b00790

20. Direct aqueous synthesis of cyanomethyl thioglycosides from reducing sugars; ready access to reagents for protein glycosylation, Stewart R. Alexandera, Antony J. Fairbanks, Org. Biomol. Chem., doi: 10.1039/C6OB01069E

21. Dual targeting of intracellular pathogenic bacteria with a cleavable conjugate of kanamycin and an antibacterial cellpenetrating peptide, A. Brezden, M.F. Mohamed, M. Nepal, J.S. Harwood, J. Kuriakose, M.N. Seleem, J. Chmielewski, J. Am. Chem. Soc., doi: 10.1021/jacs.6b04831

22. Enzymatic synthesis of oligo(ethylene glycol)-bearing cellulose oligomers for in situ formation of hydrogels with crystalline nanoribbon network structures, T. Nohara, T. Sawada, H. Tanaka, T. Serizawa, Langmuir, doi: 10.1021/acs. langmuir.6b01635

23. Galectin- 8 enhances adhesion of multiple myeloma cells to vascular endothelium and is an adverse prognostic factor, $\mathrm{M}$. Friedel, S. André, H. Goldschmidt, H.-J. Gabius, R. SchwartzAlbiez, Glycobiology, doi: 10.1093/glycob/cww066

24. Hematopoietic-derived galectin-3 causes cellular and systemic insulin resistance, P. Li, S. Liu, M. Lu, G. Bandyopadhyay, D. Oh, T. Imamura, A.M.F. Johnson, D. Sears, Z. Shen, B. Cui, L. Kong, S. Hou, X. Liang, S. Iovino, S.M. Watkins, W. Ying, O. Osborn, J. Wollam, M. Brenner, J.M. Olefsky, Cell, doi: 10.1016/j.cell.2016.10.025

25. Light-driven highly efficient glycosylation reactions, R.-Z. Mao, D.-C. Xiong, F. Guo, Q. Li, J. Duan, X.-S. Ye, Org. Chem. Front., doi: 10.1039/C6QO00021E

26. Quantitative investigation of human cell surface N-glycoprotein dynamics, H. Xiao, R. Wu, Chem. Sci., doi: 10.1039/ C6SC01814A

27. Reaction mechanisms in carbohydrate-active enzymes: Glycoside hydrolases and glycosyltransferases. Insights from $a b$ Initio quantum mechanics/molecular mechanics dynamic simulations, A. Ardèvol, C. Rovira, J. Am. Chem. Soc., doi: 10.1021/jacs.5b01156

28. Real-time NMR Studies of oxyamine ligations of reducing carbohydrates under equilibrium conditions, O.R. Baudendistel, D.E. Wieland, M.S. Schmidt, V. Wittmann, Chem. Eur. J., doi: 10.1002/chem.201603369

29. Selective recognition of $\mathrm{D}$-aldohexoses in water by boronic acid-functionalized, molecularly imprinted cross-linked micelles, J.K. Awino, R.W. Gunasekara, Y. Zhao, J. Am. Chem. Soc., doi: 10.1021/jacs.6b04613

30. Sequential one-pot multienzyme chemoenzymatic synthesis of glycosphingolipid glycans, H. Yu, Y. Li, J. Zeng, V. Thon, D.M. Nguyen, T. Ly, H.Y. Kuang, A. Ngo, X. Chen, J. Org. Chem., doi: 10.1021/acs.joc.6b01905

31. Site-directed glycosylation of peptide/protein with homogeneous O-linked eukaryotic N-glycans, Z. Wu, K. Jiang, H. Zhu, C. Ma, Z. Yu, L. Li, W. Guan, Y. Liu, H. Zhu, Y. Chen, S. Li, J. Li, J. Cheng, L. Zhang, P.G. Wang, Bioconjugate Chem., doi: 10.1021/acs.bioconjchem.6b00385

32. Small-molecule carbohydrate-based immunostimulants, C.H. Marzabadi1, R.W. Franck, Chem. Eur. J., doi: 10.1002/ chem.201601539

33. Stereoinversion of stereocongested carbocyclic alcohols via triflylation and subsequent treatment with aqueous $N, N$-dimethylformamide, H. Ochiai, T. Niwa, T. Hosoya, Org. Lett., doi: 10.1021/acs.orglett.6b02675

34. Stereoselective construction of $\beta$-mannopyranosides by anomeric O-alkylation: Synthesis of the trisaccharide core of Nlinked glycans, H. Nguyen, D. Zhu, X. Li, J. Zhu, Angew. Chem. Int. Ed., doi: 10.1002/anie.201600488

35. Stereoselective $\beta$-mannosylation by neighboring-group participation, H. Elferink, R.A. Mensink, P.B. White, T.J. Boltje, Angew. Chem. Int. Ed., doi: 10.1002/anie.201604358

36. Sugar synthesis from $\mathrm{CO}_{2}$ in Escherichia coli, N. Antonovsky, S. Gleizer, E. Noor, Y. Zohar, E. Herz, U. Barenholz, L. Zelcbuch, S. Amram, A. Wides, N. Tepper, D. Davidi, Y. Bar-On, T. Bareia, D.G. Wernick, I. Shani, S. Malitsky, G. Jona, A. Bar-Even, R. Milo, Cell, doi: 10.1016/j.cell.2016.05.064

37. Synthesis of 2-thiocarbohydrates and their binding to concanavalin A, P. Pavashe, E. Elamparuthi, C. Hettrich, H.M. Möller, T. Linker, J. Org. Chem., doi: 10.1021/acs. joc. 6 b00987

38. Synthesis of D-galactosamine and D-allosamine derivatives via a microwave-assisted preparation of 1,6-anhydroglucosamine, V.M. Dhurandhare, Y.-S. Wen, S.D. Gawande, P.-H. Liao, C.-C. Wang, J. Org. Chem., doi: 10.1021/acs. 
joc. 6 b02038

39. The hidden conformation of Lewis $\mathrm{x}$, a human histo-blood group antigen, is a determinant for recognition by pathogen lectins, J. Topin, M. Lelimousin, J. Arnaud, A. Audfray, S. Pérez, A. Varrot, A. Imberty, ACS Chem. Biol., doi: 10.1021/ acschembio.6b00333

40. The NEU1-selective sialidase inhibitor, C9-butyl-amideDANA, blocks sialidase activity and NEU1-mediated bioactivities in human lung in vitro and murine lung in vivo, S.W. Hyun, A. Liu, Z. Liu, A.S. Cross, A.C. Verceles, S. Magesh, Y. Kommagalla, C. Kona, H. Ando, I.G. Luzina, S.P. Atamas, K.H. Piepenbrink, E.J. Sundberg, W. Guang, H. Ishida, E.P. Lillehoj, S.E. Goldblum, Glycobiology, doi: 10.1093/glycob/ cww060

41. The pseudouridine synthases proceed through a glycal intermediate, G.R. Veerareddygari, S.K. Singh, E.G. Mueller, J.
Am. Chem. Soc., doi: 10.1021/jacs.6b04491

42. Total synthesis of the complete protective antigen of Vibrio cholerae O139, S.E. Soliman, P. Kováč, Angew. Chem. Int. Ed., doi: 10.1002/anie.201606116

43. XBP1s links the unfolded protein response to the molecular architecture of mature N-glycans, M.B. Dewal, A.S. DiChiara, A. Antonopoulos, R.J. Taylor, C.J. Harmon, S.M. Haslam, A. Dell, M.D. Shoulders, Chem. Biol., doi: 10.1016/ j.chembiol.2015.09.006

We hope our page will become more visible and will help researchers and students to find interesting glyco-related research more easily.

\section{Editorial Office, Osamu Kanie}

